\title{
The Effect of Anisotropy on the Impedance and Electric Field Distribution in Deep Brain Stimulation
}

Teresa Nordin, Karin Wårdell and Johannes D. Johansson

\section{Conference article}

Cite this conference article as:

Nordin, T., Wårdell, K., D., J. The Effect of Anisotropy on the Impedance and Electric Field Distribution in Deep Brain Stimulation, In Jarm;, T. (eds),8th European Medical and Biological Engineering Conference, Springer; 2020, pp. 1069-1077. ISBN: 9783030646097

DOI: https://doi.org/10.1007/978-3-030-64610-3 120

FMBE Proceedings, 1680-0737, No. 80

Copyright: Springer

http://www.springer.com/

Copyright: Springer

The self-archived postprint version of this conference article is available at Linköping University Institutional Repository (DiVA):

http://urn.kb.se/resolve?urn=urn:nbn:se:liu:diva-171873 


\title{
The Effect of Anisotropy on the Impedance and Electric Field Distribution in Deep Brain Stimulation
}

\author{
Teresa Nordin ${ }^{10000-0002-1641-9848] 凶}$, Karin Wårdell ${ }^{10000-0002-0012-7867]}$ and Johannes D Jo- \\ hansson ${ }^{10000-0003-4910-0291]}$ \\ ${ }^{1}$ Department of Biomedical Engineering, Linköping University, Sweden \\ teresa.nordin@liu.se
}

\begin{abstract}
Deep brain stimulation (DBS) is an intervention used for several neurological conditions such as Parkinson's disease. To evaluate the clinical response in relation to anatomical location, electric field simulation using the finite element method is commonly used. The models presented in different studies are varying in complexity and this study aims to evaluate the effect of including anisotropy in the tissue model using homogenous tissue with varying level of anisotropy both parallel and perpendicular to the DBS lead. As a benchmark, data from one patient was included and simulations was performed in zona incerta ( $\mathrm{Zi})$ and the internal capsule (IC). The parameters investigated were impedance, volume within the $0.2 \mathrm{~V} / \mathrm{mm}$ isosurface, radial and longitudinal expansion as well as visual representation of the isosurface. The investigations show that both the impedance and volume are increasing with increasing anisotropy together with the electric field isosurface in the principal direction of the anisotropy. When comparing different stimulation modes, current control (CC) stimulation had a steeper increase with increasing anisotropy for all parameters compared to voltage control (VC) stimulation. This could be due to a joint effect of the anisotropy and the increasing impedance. The result from the patient simulations are in the anisotropy range where simulations from the homogenous models starts to have a higher slope for all parameters. This indicates that including anisotropy in computer models will be of importance in areas of high anisotropy.
\end{abstract}

Keywords: Electric field simulation, Finite element method (FEM), Deep brain stimulation (DBS), anisotropy, impedance.

\section{Background}

Deep brain stimulation (DBS) is an established therapy for several movement disorders and is increasingly used for different psychiatric disorders and pain conditions [1]. New target areas are continuously suggested followed by evaluation of the benefit in relation to the implantation position. Electric field simulation using the finite element method is one commonly used approach for spatial evaluation where the presented models are varying in level of complexity from completely homogenous isotropic brain conductivity models to heterogenous anisotropic conductivity models [2-4]. Measurements of the conductivity indicate a nine to ten times higher conductivity along neuronal axons 
compared to the perpendicular direction [5], which indicates an importance of including the anisotropy in tissue conductivity modelling. Impedance and light reflectance have been measured along the trajectory for DBS implantation and indicated that the impedance is increasing in the internal capsule (IC) [6], an area with highly condensed fiber tracts, which would impact computer simulations especially for current control (CC) stimulation. Previous study evaluating implantation in zona incerta (Zi) showed only small difference when extending the tissue model with anisotropy [7]. However, the level of anisotropy in the stimulated area is one important parameter to consider when evaluating the effect of an anisotropic model.

This study aims to investigate how the level of anisotropy affects the simulation result for voltage control (VC) and CC stimulation using homogenous tissue models and a patient example for benchmarking of expected result in a brain.

\section{Material and Methods}

All investigations in this project were made based on the geometry of a conventional quadrupolar DBS lead with cylindrical contacts (3389, Medtronic Inc, USA), where the most distal contact was used as active contact with amplitude $1 \mathrm{~V} / 1 \mathrm{~mA}(\mathrm{VC} / \mathrm{CC})$. The electric field distribution was computed with the finite element method using the equation of continuity for steady currents (Comsol Multiphysics v. 5.4, Sweden) (Eq. 1)

$$
\nabla \cdot \boldsymbol{J}=-\nabla \cdot(\sigma \nabla V)=0
$$

where $\mathbf{J}$ is the current density $\left(\mathrm{A} / \mathrm{m}^{2}\right), \sigma$ is the electrical conductivity $(\mathrm{S} / \mathrm{m})$ and $V$ is the electrical potential $(\mathrm{V})$. During simulation, all inactive contacts were set to floating potential, plastic part of the lead to electrical insulation and the outer bounding box to ground. For comparison of the spatial area of impact, an electric field isolevel of $0.2 \mathrm{~V} / \mathrm{mm}$ was used corresponding to activating neurons of approximately $3-4 \mu \mathrm{m}$ in diameter $[4,8,9]$.

\subsection{Brain Tissue Models}

To investigate the effect of anisotropy, a brain conductivity model was constructed consisting of homogenous tissue modelled as a box of $100 \times 100 \times 80 \mathrm{~mm}^{3}$. The tissue conductivity was defined using a conductivity tensor, $\sigma$, represented as a diagonal matrix and adjustment of the diagonal elements generated variable level of anisotropy parallel and perpendicular to the lead (Eq. 2),

$$
\boldsymbol{\sigma}=\left[\begin{array}{ccc}
\sigma_{x x} & 0 & 0 \\
0 & \sigma_{y y} & 0 \\
0 & 0 & \sigma_{z z}
\end{array}\right], \begin{cases}\sigma_{z z}=(1+2 \alpha) \sigma_{i s o}, & \sigma_{y y}=\sigma_{x x}=(1-\alpha) \sigma_{i s o}, \text { anisotropy } \| \text { lead } \\
\sigma_{y y}=(1+2 \alpha) \sigma_{i s o}, & \sigma_{z z}=\sigma_{x x}=(1-\alpha) \sigma_{i s o} \text {, anisotropy } \perp \text { lead }\end{cases}
$$

where, $\sigma_{\text {iso }}$, is representing corresponding isotropic conductivity based on tabulated brain tissue values (white matter $(\mathrm{WM}): \sigma_{\mathrm{WM}}=0.0754$, gray matter $(\mathrm{GM}): \sigma_{\mathrm{GM}}=0.123$ ) and $\alpha$ is a parameter used to scale the anisotropy $(\alpha \in[-0.4,0.8]$, step size 0.1$)$. 
For comparison, a patient assigned to DBS therapy in Zi at the Department of Neurosurgery at Linköping University Hospital was included in the study. The patient gave informed written consent and the study was approved by the Local Ethics Committee (No. 2012/434-31 and 2018/143-32). Preoperative $T_{2}$ MRI (Ingenia 3T, Philips Healthcare, the Netherlands) was used for intensity-based tissue segmentation and conductivity assignment $\left(\sigma_{\text {iso }}\right)$ according to previous work $[2,10]$. The anisotropic conductivity was estimated based on preoperative diffusion MRI according to Eq. 3,

$$
\boldsymbol{\sigma}=\frac{\sigma_{e}}{d_{e}} \cdot \boldsymbol{D}
$$

where $\boldsymbol{D}$ is the diffusion tensor, $\sigma_{e}$ is the effective extracellular conductivity, estimated as $\sigma_{\text {iso, }}$ and $d_{e}$ is the effective extracellular diffusivity, estimated as trace(D)/3 [7]. Simulations were made both in $\mathrm{Zi}$, according to patient stereotactic implantation coordinates, and by using a theoretical position in IC to represent a brain region with high anisotropy.

\subsection{Data Analysis}

For all simulations, the impedance, calculated using the contact voltage divided by contact current, and volume within the $0.2 \mathrm{~V} / \mathrm{mm}$ isosurface were extracted. To evaluate the shape of the isosurface, visual inspection was performed along with distance measures of maximum radial distance $\left(d_{\mathrm{rmax}}\right)$, minimum radial distance $\left(d_{\mathrm{rmin}}\right)$ and longitudinal distance $\left(d_{1}\right)$. The maximum and minimum radial distance were computed in the plane perpendicular to the lead at the height of maximal isosurface extension. For the anisotropy direction parallel to the lead, the radial distance is presented as the mean of the max and min distance $\left(d_{\mathrm{r}}\right)$ and for anisotropy perpendicular to the lead, radial distance is presented as radial distance in $\mathrm{x}$-direction $\left(d_{\mathrm{rx}}\right)$ and $\mathrm{y}$-direction $\left(d_{\mathrm{ry}}\right)$. For the depiction of the different distance measures see Fig $3 \mathrm{c}$. All measures are compared to the level of anisotropy using the fractional anisotropy (FA).

\section{$3 \quad$ Result}

Simulations $(n=105)$ were computed using homogenous tissue with variable level of anisotropy. These were compared to patient-specific simulations in $\mathrm{Zi}(n=2)$ which have a mid-range level of anisotropy and IC $(n=2)$ with a higher level of anisotropy.

\subsection{Impedance}

The impedance of the model is increasing with the level of anisotropy (range for GM: 884-2003 $\Omega$, WM:1442-2574 $\Omega$ ) with lowest impedance at $\alpha=0 \pm 0.1$, result independent of stimulation mode, Fig 1a. As the impedance is inversely related to the conductivity, lower conductivity scales the curves to higher impedance. Comparing anisotropy parallel and perpendicular to the lead, similar curve patterns are seen but parallel anisotropy direction have a slightly higher slope for the highest anisotropy values. The 
patient-specific simulations gave an impedance of $1204 \Omega$ in $\mathrm{Zi}$ and $1432 \Omega$ in IC. The FA within the isosurface was $0.51 \pm 0.05$ and $0.68 \pm 0.04(m \pm s d)$ for $\mathrm{Zi}$ and IC respectively, which corresponds to an $\alpha$ of approximately \pm 0.3 and \pm 0.5 , placing these datapoints between the grey and white matter curves.

\subsection{Volume}

For VC stimulation, the volume within the isosurface and all the distance measures are independent of the conductivity for homogenous tissue. Using CC stimulation, the different direction of anisotropy generated highly similar result. For CC, the lowest volume is achieved for $\alpha=0$ (isotropic), while for $\mathrm{VC}$ the lowest volume is achieved at $\alpha=-0.1$ and $\alpha=0.3$ for anisotropy in the perpendicular and parallel direction respectively Fig $1 b$.

The shape of the isosurface is clearly affected by the anisotropy where a higher anisotropy will extend the electric field in that direction, see Fig. 2.

a)

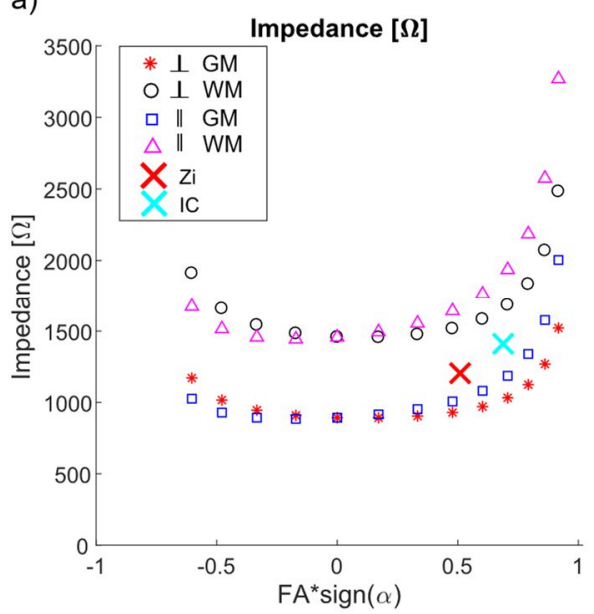

b)

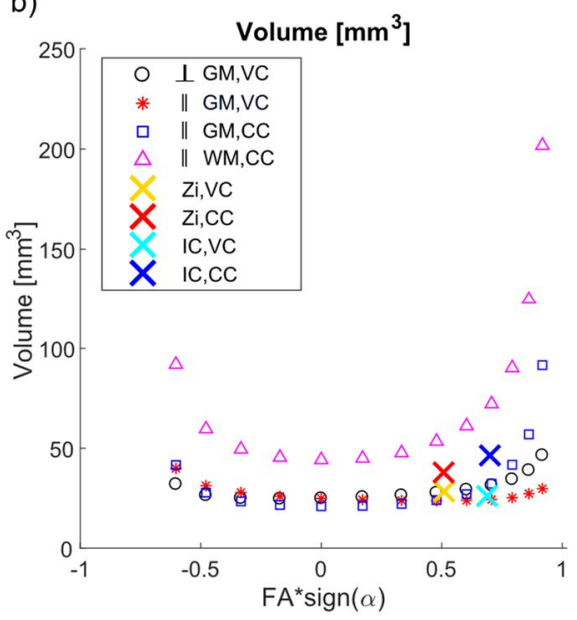

Fig. 1. Impedance (a) and volume (b) results from the simulations. FA=Fractional anisotropy, $G M=$ grey matter, WM=white matter, $\perp$ and $\|$ direction of anisotropy compared to lead, $V C=$ voltage control, $C C=$ current control, $Z i=$ zona incerta, $I C=$ internal capsule.

\subsection{Distance Measures}

All distance measurements can be found in Fig. 3.

Radial Distance. For anisotropy parallel to DBS lead, $d_{\mathrm{r}}$ is continuously decreasing with increasing $\alpha$ in $\mathrm{VC}$ mode and increasing with increasing anisotropy for CC stimulation, with lowest radial distance at $\alpha=0.1$ and $\alpha=0.2$ for GW and WM respectively (range: $1.65-3.37 \mathrm{~mm}$ ).

For anisotropy perpendicular to the lead, $d_{\mathrm{ry}}$ is increasing with increasing $\alpha$ in both stimulation modes (range: $1.55-6.27 \mathrm{~mm}$ ). The $d_{\mathrm{rx}}$ is decreasing with increasing $\alpha$ in 
VC mode. For CC stimulation, $d_{\mathrm{rx}}$ is increasing with increasing anisotropy (range: 1.75$3.48 \mathrm{~mm}$ ), with lowest radial distance for $\alpha=0.2$. All radial distance curves have a rather flat curve from $\alpha=-0.2$ to 0.3 .

Investigating the patient example, the maximal radial distance is affected to a very small extent (VC: $\Delta d_{\text {rmax }}=-0.09 \mathrm{~mm}, \mathrm{CC}: \Delta d_{\mathrm{rmax}}=0.03 \mathrm{~mm}$ ), but the minimal radial distance is slightly more effected (VC: $\Delta d_{\mathrm{rmin}}=-0.22 \mathrm{~mm}, \mathrm{CC}: \Delta d_{\mathrm{rmin}}=-0.17 \mathrm{~mm}$ ), where $\Delta d=d(\mathrm{IC})-d(\mathrm{Zi})$.

Longitudinal Distance. When varying the anisotropy parallel to the lead, $d_{1}$ is increased with increasing $\alpha$ for both VC and CC (range: 2.96-11.14 mm).

For anisotropy variation perpendicular to the lead, $d_{1}$ is decreased with increasing $\alpha$ in VC mode but with very small variation at $\alpha>-0.3$, giving a maximum difference of $0.17 \mathrm{~mm}$. For CC stimulation, $d_{1}$ is very similar for $\alpha=-0.1$ to 0.5 , lowest point $\alpha=0.2$, but then increasing with higher anisotropy (range: $3.13-6.23 \mathrm{~mm}$ ).

Comparing with the patient example, the $d_{1}$ is differently affected for the different stimulation modes (VC: $\Delta d_{1}=0.09 \mathrm{~mm}, \mathrm{CC}: \Delta d_{1}=0.67 \mathrm{~mm}$ ).

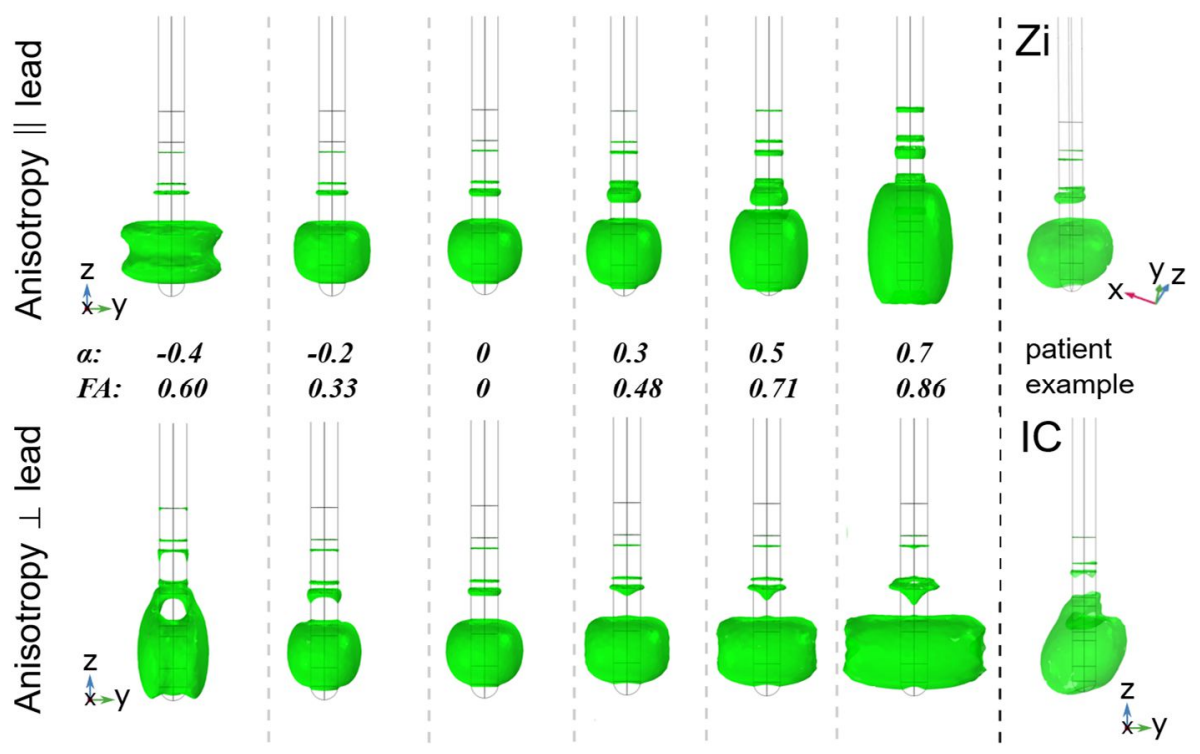

Fig. 2. Visualization of the difference in $0.2 \mathrm{~V} / \mathrm{mm}$ isosurface shape for different level of anisotropy. Increasing level of anisotropy parallel and perpendicular to the DBS lead is shown in top and bottom row respectively, compared with the patient examples to the right. All images are from current control stimulation with grey matter tissue, similar patterns were found for all simulations. Note that negative $\alpha$ gives a lower eigenvalue for the investigated direction and thereby the isosurface is compressed in that direction while it expands further out in the other two directions. 
a)

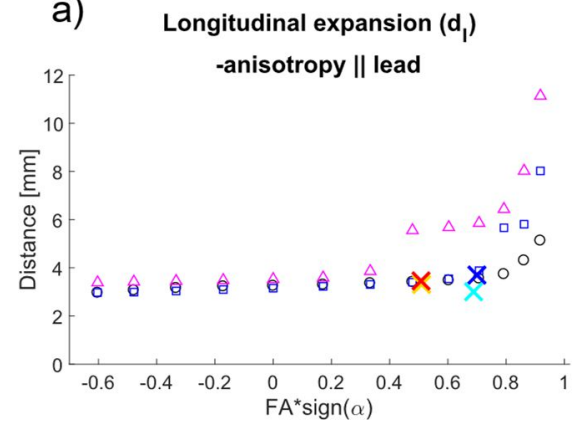

b)

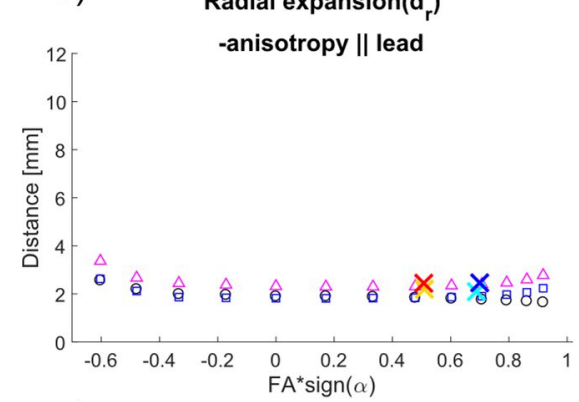

c)

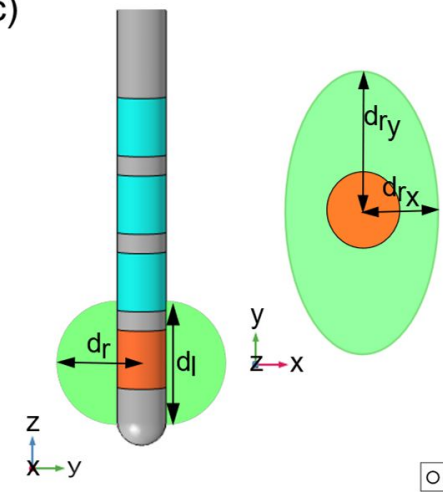

d) Longitudinal expansion $\left(d_{1}\right)$

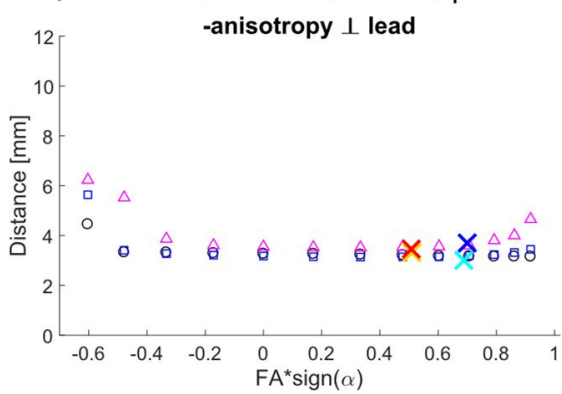

e) Radial expansion in y-direction $\left(d_{r_{y}}\right)$

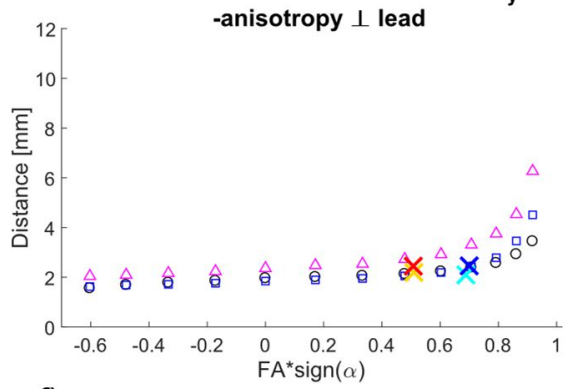

f) Radial expansion in $\mathrm{x}$-direction $\left(\mathrm{d}_{\mathrm{r}_{\mathrm{x}}}\right)$

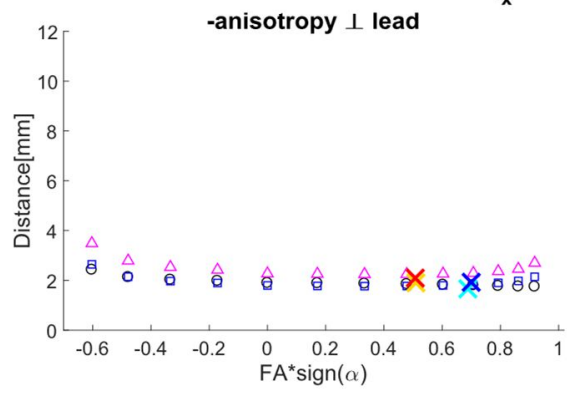

$O G M, V C \square G M, C C \triangle W M, C C \times Z i, V C \times Z i, C C \times I C, V C \times I C, C C$

Fig. 3. Distance measures of the $0.2 \mathrm{~V} / \mathrm{mm}$ isosurface. For anisotropy parallel to lead a) longitudinal expansion $\left(d_{1}\right)$ and b) radial expansion $\left(d_{\mathrm{r}}\right)$ compared with patient data of $\left.d_{\mathrm{rmax}}, \mathrm{c}\right)$ depictions of the distance measures. For anisotropy perpendicular to lead d) longitudinal expansion $\left(d_{1}\right)$, e) radial expansion in y-direction $\left(d_{\mathrm{ry}}\right)$ compared with patient data of $d_{\mathrm{rmax}}$ and f) radial expansion in x-direction $\left(d_{\mathrm{rx}}\right)$ compared with patient data of $d_{\mathrm{rmin}}$. GM=grey matter, WM=white matter, $V C=$ voltage control, $C C=$ current control, $Z i=$ zona incerta, $I C=$ internal capsule 


\section{Discussion}

The result shows that if the anisotropy is increased in one direction the impedance will also increase (Fig. 1), supporting the theory based on previous study [6] where IC had a notably higher impedance than subcortical white matter. A small difference can also be seen by the direction of the anisotropy, but largest impact has the tissue conductivity, which is inversely related to the impedance, especially for low levels of anisotropy.

In general, higher anisotropy will increase the volume within the isosurface and the current spread in the tissue anisotropy direction. However, for high anisotropy levels, this increase is much steeper for current control stimulation compared to voltage control. This could be due to a joint effect of the anisotropy and increase of the impedance, since higher impedance requires higher voltage for the same current. Followingly it also implies that $\mathrm{CC}$ stimulation is more sensitive to tissue variations when applying this methodology with electric field isolevel.

In relation to patient stimulation, if implantation is done parallel to the fiber tract, the radial distance is the parameter of highest importance. This distance is affected by the anisotropy (decreasing for $\mathrm{VC}$ and increasing for $\mathrm{CC}$ ) which might affect the required amplitude. The difference in radial distance between $\mathrm{FA}=0.86$ and $\mathrm{FA}=0$ is approximately $0.2 \mathrm{~mm}$ which corresponds to an amplitude change of approximately $0.2-0.3 \mathrm{~V} / \mathrm{mA}$.

All investigated parameters have a low variation for $\mathrm{FA}<0.5$ but an increasing slope for higher FA values, Fig 1 and 3. The level of anisotropy in the patient-specific simulations is slightly above FA $=0.5$ which implies that an anisotropic model will impact the result for brain areas with very high anisotropy. However, for areas with lower anisotropy, the effect is small and the conductivity estimation is of higher importance, especially for CC stimulation. One important aspect is that several studies use an encapsulation layer around the DBS lead which is usually modelled as bulk tissue [11,12]. Using that without including information of anisotropy will reduce the effect of the anisotropy which probably should be compensated for, especially if the simulation is made in a highly anisotropic area.

Worth noting is that a conductivity relationship of 10:1 parallel compared to perpendicular to neuronal tract corresponds to an FA value of 0.89 . The mean FA within the isosurface of IC stimulation was 0.68 which is notably lower. This could be due to fibers not being completely parallel or a mixed tissue effect, where the isosurface span further out and included more tissue regions. Another possibility is that the conductivity tensor based on diffusion MRI is not as anisotropic as expected based on measurements, which could be a topic for future investigation.

Many studies, both on DBS and electroencephalography computations, have investigated the effect of adding anisotropy to the tissue model by using diffusion tensor datasets $[3,7,11,13-15]$. However, as far as the authors know no one have done any evaluation regarding the impact when varying the level of anisotropy. The result from previous studies are also varying where some find that the anisotropy is of high importance $[3,11]$ and some reports only small differences when comparing isotropic and anisotropic brain models [7,13-15]. The reason for the different result could be the use of different models and parameters for evaluation. Another aspect shown by this work 
is that given the variability of anisotropy in the brain, the result will be affected by the area of choice where some areas might display a high impact of the anisotropic features where other areas will only display a small impact.

\section{Conclusion}

When evaluating the level of anisotropy for DBS electric field simulations it is evident that both impedance and isosurface shape is impacted by the anisotropy. However, dependent of the stimulation mode and the tissue composition, adding anisotropy to the tissue model can be of variable importance and aspects as tissue conductivity and heterogeneity have a higher impact on the result in low anisotropy areas. This further stress the importance of using a representative model and knowledge regarding when simplifications can be appropriate.

Acknowledgement. The authors would like to thank Peter Zsigmond for patient data collection. This project was supported by the Swedish Foundation for Strategic Research (SSF BD15-0032) and Swedish Research Council (VR 2016-03564).

\section{Conflict of Interest. None}

\section{References}

1. Lozano, A.M., Lipsman, N., Bergman, H., Brown, P., Chabardes, S., Chang, J.W., Matthews, K., McIntyre, C.C., Schlaepfer, T.E., Schulder, M., Temel, Y., Volkmann, J., Krauss, J.K.: Deep brain stimulation: current challenges and future directions. Nature Reviews Neurology 15(3), 148-160 (2019). doi:10.1038/s41582-018-0128-2

2. Åström, M., Zrinzo, L.U., Tisch, S., Tripoliti, E., Hariz, M.I., Wårdell, K.: Method for patient-specific finite element modeling and simulation of deep brain stimulation. Medical \& Biological Engineering \& Computing 47(1), 21-28 (2009). doi:10.1007/s11517-008-0411-2

3. Howell, B., McIntyre, C.C.: Analyzing the Tradeoff between Electrical Complexity and Accuracy in Patient-Specific Computational Models of Deep Brain Stimulation. Journal of neural engineering 13(3), 036023-036023 (2016). doi:10.1088/1741-2560/13/3/036023

4. Mädler, B., Coenen, V.A.: Explaining Clinical Effects of Deep Brain Stimulation through Simplified Target-Specific Modeling of the Volume of Activated Tissue. American Journal of Neuroradiology 33(6), 1072-1080 (2012). doi:10.3174/ajnr.A2906

5. Nicholson, P.W.: Specific impedance of cerebral white matter. Experimental Neurology 13(4), 386-401 (1965). doi:10.1016/0014-4886(65)90126-3

6. Johansson, J.D., Blomstedt, P., Haj-Hosseini, N., Bergenheim, A.T., Eriksson, O., Wårdell, K.: Combined Diffuse Light Reflectance and Electrical Impedance Measurements as a Navigation Aid in Deep Brain Surgery. Stereotactic and Functional Neurosurgery 87(2), 105 113 (2009). doi:10.1159/000202977 
7. Nordin, T., Zsigmond, P., Pujol, S., Westin, C.-F., Wårdell, K.: White matter tracing combined with electric field simulation - A patient-specific approach for deep brain stimulation. NeuroImage: Clinical 24, 102026 (2019). doi:10.1016/j.nicl.2019.102026

8. Åström, M., Diczfalusy, E., Martens, H., Wårdell, K.: Relationship between Neural Activation and Electric Field Distribution during Deep Brain Stimulation. IEEE Transactions on Biomedical Engineering 62(2), 664-672 (2015). doi:10.1109/TBME.2014.2363494

9. Kuncel, A.M., Cooper, S.E., Grill, W.M.: A method to estimate the spatial extent of activation in thalamic deep brain stimulation. Clinical neurophysiology : official journal of the International Federation of Clinical Neurophysiology 119(9), 2148-2158 (2008). doi:10.1016/j.clinph.2008.02.025

10. Johansson, J.D., Alonso, F., Wårdell, K.: Patient-Specific Simulations of Deep Brain Stimulation Electric Field with Aid of In-house Software ELMA. In: 2019 41st Annual International Conference of the IEEE Engineering in Medicine and Biology Society (EMBC), pp. 5212-5216. IEEE, Berlin, Germany (2019). doi: 10.1109/EMBC.2019.8856307

11. Chaturvedi, A., Butson, C.R., Lempka, S.F., Cooper, S.E., McIntyre, C.C.: Patient-specific models of deep brain stimulation: Influence of field model complexity on neural activation predictions. Brain Stimulation 3(2), 65-77 (2010). doi:10.1016/j.brs.2010.01.003

12. Alonso, F., Latorre, M.A., Göransson, N., Zsigmond, P., Wårdell, K.: Investigation into Deep Brain Stimulation Lead Designs: A Patient-Specific Simulation Study. Brain Sciences 6(3), 39 (2016). doi:10.3390/brainsci6030039

13. Rullmann, M., Anwander, A., Dannhauer, M., Warfield, S.K., Duffy, F.H., Wolters, C.H.: EEG source analysis of epileptiform activity using a $1 \mathrm{~mm}$ anisotropic hexahedra finite element head model. NeuroImage 44(2), 399-410 (2009). doi:10.1016/j.neuroimage.2008.09.009

14. Schmidt, C., Rienen, U.v.: Modeling the Field Distribution in Deep Brain Stimulation: The Influence of Anisotropy of Brain Tissue. IEEE Transactions on Biomedical Engineering 59(6), 1583-1592 (2012). doi:10.1109/TBME.2012.2189885

15. Åström, M., Lemaire, J.-J., Wårdell, K.: Influence of heterogeneous and anisotropic tissue conductivity on electric field distribution in deep brain stimulation. Medical \& Biological Engineering \& Computing 50(1), 23-32 (2012). doi:10.1007/s11517-011-0842-z 